\title{
Maximum Metallic Conductivity in Si-MOS Structures
}

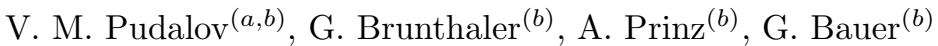 \\ ${ }^{(a)}$ P. N. Lebedev Physical Institute of the Russian Academy of Sciences, Moscow, Leninsky prosp. 53, Russia \\ (b) Institut für Halbleiterphysik, Johannes Kepler Universität, Linz, A-4040, Austria
}

(July 8, 2021)

\begin{abstract}
We found that the conductivity of the two-dimensional electron system in Si-MOS structures is limited to a maximum value, $G_{\max }$, as either density increases or temperature decreases. This value $G_{\max }$ is weakly disorder dependent and ranging from 100 to $140 e^{2} / h$ for samples whose mobilities differ by a factor of 4 .
\end{abstract}

PACS numbers: 71.30.+h, 72.15.Rn, 73.40.Qv

According to the conventional theory of metals [i], the conductivity of the two-dimensional carrier system should vanish in the limit of zero temperatures. Recently, an unconventional metallic-like temperature dependence of the conductivity was found in two-dimensional (2D) carrier systems in different materials [2,3]. The effect manifests itself in the exponentially strong rise of the conductivity $G$ (by about one order of magnitude in Si-MOS structures where it is most pronounced 22]) as the temperature decreases below $\sim 0.3 E_{F} / k_{B}$ 位. The origin of the effect remains under discussion $[5]$ and is intimately related to a question on the ground state conductivity in the $T=0$ limit. The existing experiments are taken at finite temperatures (though much less than $E_{F} / k_{B}$ ) and it is not absolutely clear whether or not the observed "metallic-like" temperature behavior of $G$ corresponds to the ground state conductivity. Since for the Fermi liquid the only possibility is $G=0$, it was suggested, that the two-dimensional strongly interacting carrier system can become a perfect metal with infinite conductivity $G$, at $T=0$ [6] but exhibiting non-Fermi-liquid behavior. It was even suggested that the $2 \mathrm{D}$ interacting system could become a superconductor [7].

In order to verify these possibilities, we have extended the measurements to carrier densities about 100 times higher than the critical conductivity $n_{\mathrm{c}}$, at which the exponential decrease of the resistivity sets in [2,3]. Our investigations are motivated by the fact that as density increases, the Drude conductivity increases and "disorder" $\left(1 / k_{F} l\right)$ decreases. From the measurements at high density, we expected to verify whether or not the metallic like conductivity survives at high $G$ values, to probe the role of Coulomb interaction effects (where the ratio of the Coulomb to Fermi energy decreases proportionally to $n^{-1 / 2}$ ) and of spin-related effects (which should persist as density increases).

We have found that the conductivity in (100) Si-MOS structures shows a maximum as a function of carrier density. The maximum value, $G_{\max } \approx 100-140$ is weakly dependent on the mobility of the sample (conductivity throughout this paper is in units of $e^{2} / h=$ $1 / 25813 \mathrm{Ohm}^{-1}$, and the resistivity $\left.\rho=1 / G\right)$. The strong exponential dependence of $G(T)$ (with $d G / d T<$ $0)$ which exists at relatively high temperatures $T \leq$ $0.3 E_{F} / k_{B}$ was found to persist up to the highest density studied. However, at low temperatures, $T<$ $0.007 E_{F} / k_{B}$ (and at least for high densities), in the vicinity of $n=n_{\max }$, this metallic like dependence transforms into a weak $\ln T$ dependence with a positive derivative, $d G / d T>0$, thus indicating the onset of a weakly localized state.

The ac- and dc-measurements of the conductivity were performed on (100) Si-MOS structures at low dissipated power. Five samples were studied in the density range 0.8 to $100 \times 10^{11} \mathrm{~cm}^{-2}$; their relevant parameters are listed in Table 1. In order to adjust the biasing current such as not to destroy the phase coherence in the carrier system, we determined the phase breaking time, $\tau_{\phi}$, from the weak negative magnetoresistance [8] in low magnetic fields. Measurements were taken in the temperature range 0.29 to $45 \mathrm{~K}$, and, partly, 0.018 to $4 \mathrm{~K}$, by sweeping slowly the temperature during several hours. The data taken on all five samples were qualitatively similar.

A typical density dependence of the conductivity in the "metallic" range, $n=(6-100) \times 10^{11} \mathrm{~cm}^{-2}$, is shown in Fig. 1 for different temperatures, 0.3 to $41 \mathrm{~K}$. The conductivity, $G$, first increases with density, reaches a maximum at $n=(35-43) \times 10^{11} \mathrm{~cm}^{-2}$, and then decreases again. Shubnikov-de Haas data taken on a few high mobility samples show the onset of a second frequency at $n \geq 55 \times 10^{11} \mathrm{~cm}^{-2}$, which is due to population of the second subband. The reversal of the density dependence of the conductivity may be caused by an increase of the scattering rate as $E_{F}$ approaches the bottom of the next subband. Table I shows that the maximum conductivity value is weakly dependent on disorder, $G=100-140$ for the studied samples. At the same time, the density values $n_{\max }$, corresponding to the maximum conductivity, increase by a factor 2 as the mobility decreases by a factor 4.

In Fig. 2, the temperature dependence of the conductivity is shown for high densities, $(8-80) \times 10^{11} \mathrm{~cm}^{-2}$. As density increases, the conductivity, first increases (the curves 1 to 6 ), reaches a maximum (the curve 6 ) at a 
density $n_{\max }$ (which is $32 \times 10^{11} \mathrm{~cm}^{-2}$ for Si-15a), and, finally, decreases with density (curves 7 - 12). This leads to a crossing of the $G(T)$ - curves taken at different densities $n>n_{\max }$. Such a crossing has also been reported to occur for $p$-GaAs/AlGaAs in Ref. [9]. However, in our measurements, the $G(n)$ curves for different temperatures, do not intercept at a single density.

In Fig. 2, the triangles depict for each curve the temperature $T^{*}=0.007 E_{F} / k_{B}$ for the corresponding density. In the region confined between $T=0.05 E_{F} / k_{B}$ and $0.007 E_{F} / k_{B}$, the exponential dependence seems to "saturate", but in fact, it crosses over, below $\approx T^{*}=$ $0.007 E_{F} / k_{B}$, to a weaker dependence. The "high temperature behavior" (for $T>T^{*}$ ) of the conductivity remains metallic-like for all curves in Fig. 2, up to the highest density studied. However, the curves taken for high densities (close to the maximum conductance), at low temperatures clearly show the onset of a localizing $\ln T$ dependence with $d G / d T>0$. The localizing low temperature dependence is shown in an expanded scale in Fig. 3a. As the temperature is varied, it persists for one order of magnitude, and does not saturate at low temperatures. Its slope $d G / d \ln T \approx 0.35$ is consistent with the conventional theory of the weak localization [8]. Since the "low-temperature" localizing $T$-dependence develops on the background of the strong exponential increase in conductivity at "high temperatures", we conclude, that the exponential raise in $G(T)$ can not be considered as a proof of the metallic conductance, at least for high densities $n \gg n_{c}$.

The change of sign of $d G / d T$ shown in Fig. 3 a for $T<3 \mathrm{~K}$ (for $n \approx n_{\max }$ ) is not caused by significant changes in disorder for densities around $n_{\max }$. The conductance $G$ (which is $2 \times k_{F} l$ in the Drude approximation for the two valley system) is of the order of $100\left(k_{F}\right.$ is the Fermi wave vector and $l$ is the mean free path). Also, for the spin-orbit parameter in the chiral model [10], $2 \Delta \tau / \hbar \approx 4-8$ holds ( $\Delta$ is the zero magnetic field "spin-splitting" at $\left.E=E_{F}\right)$. Therefore, the above parameters seem to be not important at $n \sim n_{\max }$.

The picture is less clear for lower densities, $n \sim(1-$ $15) \times 10^{11} \mathrm{~cm}^{-2}$ (see Figs. $3 \mathrm{~b}$ and c), where the slope decreases, disappear and finally changes sign to the negative "delocalizing" one $d G / d T<0$ [1]. If the above scenario would persist to much lower temperatures, the conductivity $G(T)$ data taken for different densities would cross each other at finite temperatures (but at much lower temperature than shown here). This possibility seems to be unphysical and means that at least a part of the data taken for lowest temperatures (most probable, the lower density ones) do not correspond to the ground state conductivity. One can not exclude, therefore, that the low-temperature data may be affected by the tail of the strong metallic-like exponential "high temperature" dependence, extending down to low temperatures.

Anyhow, on the basis of the data shown, it seems rather unlikely, that the conductivity will grow to infinity in the $T \rightarrow 0$ limit, both for high as well as for low carrier densities. In order to reach a more definite conclusion, the measurements have to be taken down to temperatures $T \ll T^{*} \sim 0.007 E_{F} / k_{B}$.

In summary, we have found that the conductivity value in (100) Si-MOS structures is limited to a finite value, $G_{\max } \sim 140$ as density or temperature vary. We found that the strong metallic-like increase in the conductivity as $T$ decreases (visible at "high temperatures" $\left.T>0.01 E_{F} / k_{B}\right)$ and the "low temperature" behavior (for $T<0.01 E_{F} / k_{B}$ ) are rather independent of each other. Despite the observation that the maximum conductivity value is nearly the same for different Si-MOS samples, we do not have evidence that this value is related to a many body ground state [5]. The fact that the maximum in $G(T)$ appears at a finite temperature $\left(\sim T^{*}=0.007 E_{F} / k_{B}\right)$ indicates actually a single particle origin. Such a maximum of $G$ could be the result of a superposition of a scattering mechanism and weak localization effects. The behavior of the conductivity for lower temperatures requires further studies.

V.P. acknowledges discussions with B. Altshuler, M. Baranov, A. Finkel'stein, V. Kravtsov, S. V. Kravchenko, D. Maslov, A. Mirlin, and I. Suslov. The work was supported by RFBR 97-02-17378, by the Programs "Physics of solid-state nanostructures" and "Statistical physics", by INTAS, NWO, and by FWF P13439, Austria.

[1] E. Abrahams, P.W. Anderson, D. C. Licciordello, and T. V. Ramakrishnan, Phys. Rev. Lett. 42, 673 (1979).

[2] S. V. Kravchenko, G. V. Kravchenko, J. E. Furneaux, V. M. Pudalov, and M. D'Iorio, Phys. Rev. B 50, 8039 (1994). S. V. Kravchenko, W. E. Mason, G. E. Bowker, J. E. Furneaux, V. M Pudalov, and M. D'Iorio, Phys. Rev. B 51, 7038 (1995).

[3] D. Popović, A. B. Fowler, S. Washburn, Phys. Rev. Lett., 79, 1543 (1997). D. Popović, A. B. Fowler, S. Washburn, Physica B, 249-251 701 (1998). P. T. Coleridge, R. L. Williams, Y. Feng, and P. Zawadzki, Phys. Rev. B 56, R12764 (1997). M. D'Iorio, D. Brown, H. Lafontain, cond-mat/9708201. Y. Hanein, U. Meirav, D. Shahar, C. C. Li, D. C. Tsui, and H. Shtrikman, Phys. Rev. Lett. 80, 1288 (1998). M. Y. Simmons, A. R. Hamilton, M. Pepper, E. H. Linfield, P. D. Rose, and D. A. Ritchie, Phys. Rev. Lett. 80, 1292 (1998).

[4] V. M. Pudalov JETP Lett., 66, 170 (1997).

[5] S. Chakravarty, L. Yin, E. Abrahams, Phys. Rev. B 58 R559 (1998). C. Castellani, C. diCastro, P. A. Lee, Phys. Rev. B 57, R938 (1998). A. M. Finkel'stein, Sov. Sci. Reviews/section A- Physics Reviews, Ed. I. M. Khalatnikov, 14, 3 (1990). C. Castellani, C. DiCastro, H. Fukuyama, P. A. Lee and M. Ma, Phys. Rev. B 33, 7277 (1986). C. 
Castellani, C. DiCastro, P. A. Lee, cond-mat/9801006. S. Ckhakravarty, S. Kivelson, C. Nayak and K. Völker, cond-mat/9805383.

[6] V. Dobrosavljević, E. Abrahams, E. Miranda, and S. Chakravarty, Phys. Rev. Lett. 79, 455 (1997).

[7] P. Phillips, Y. Wan, cond-mat/9704200. P. Phillips, Y. Wan, I. Martin, S. Knysh, D. Dalidovich, condmat/9709168. Nature, 395, 253 (1998). D. Belitz, T. R. Kirkpatrick, cond-mat/9705023.

[8] For a review see: B. L. Altshuler, A. G. Aronov, in:
Electron-Electron Interaction in Disordered Systems, A. L. Efros, M. Pollak, Eds., North-Holland, Amsterdam, 1985.

[9] A. R. Hamilton, M. Y. Simmons, M. Pepper, E. H. Linfield, P. D. Rose, D. A. Ritchie,cond-mat/9808108.

[10] M. A. Skvortsov, cond-mat/9712135. Y. Lyanda-Geller, Phys. Rev. Lett. 80, 4273 (1998). I. V. Gornyi et al., JETP Lett, 68, 338 (1998).

[11] V. M. Pudalov, G. Brunthaler, A. Prinz, and G. Bauer, cond-mat/9801077 $\mathrm{v} 2$; JETP Lett. 68, 534 (1998).

TABLE I. The parameters of the studied samples. Density is in unites of $10^{11} \mathrm{~cm}^{-2}$.

\begin{tabular}{|c|c|c|c|c|c|}
\hline sample & $\mu_{\text {peak }}\left(\mathrm{m}^{2} / \mathrm{Vs}\right)$ & $n_{\mathrm{c}, 1}$ & $G_{\mathrm{c}, 1}$ & $n_{\max }$ & $G_{\max }$ \\
\hline Si-22 & 3.3 & 0.83 & 0.5 & 39.3 & 140 \\
\hline Si-15a & 3.2 & 0.82 & 0.4 & 32.1 & 133.7 \\
\hline $\mathrm{Si}-43 \mathrm{~b}$ & 1.96 & 1.4 & 1.5 & 35 & 124.5 \\
\hline
\end{tabular}

FIG. 1. Density dependence of the conductivity for the sample Si22 at 17 different temperatures, $T=0.29,1.5,3.9,4.8$, $5.5,7.9,8.5,10.5,12.5,16,19,21.5,23.5,26,32,36,41 \mathrm{~K}$. The upper arrow show the density, $n_{\max }$, corresponding to the maximum conductance, the lower arrow is for the critical density, $n_{c}$.

FIG. 2. Temperature dependence of the conductivity for Si-15a in the range 0.29 to $45 \mathrm{~K}$ at 12 density values: 1-8.10, 2- 10.3, 3- 15.7, 4- 21.2, 5- 26.6, 6- 32.1, 7- 42.94, 8- 48.4, 9- 53.8, 10- 64.7, 11- 75.6, 12- $86.5 \times 10^{11} \mathrm{~cm}^{-2}$. Continuous lines are for the densities $n<n_{\max }$, dotted lines for $n>n_{\max }$. The empty triangles depict $T^{*}$ for the dotted curves $1-5$, full triangles are for the continous curves 6 - 12.

FIG. 3. Expanded low-temperature part of the conductivity for the sample Si-43b in the range 0.29 to $10 \mathrm{~K}$ for three different density values indicated on each panel. Arrows mark the temperature $T^{*}=0.007 E_{F} / k_{B}$. 


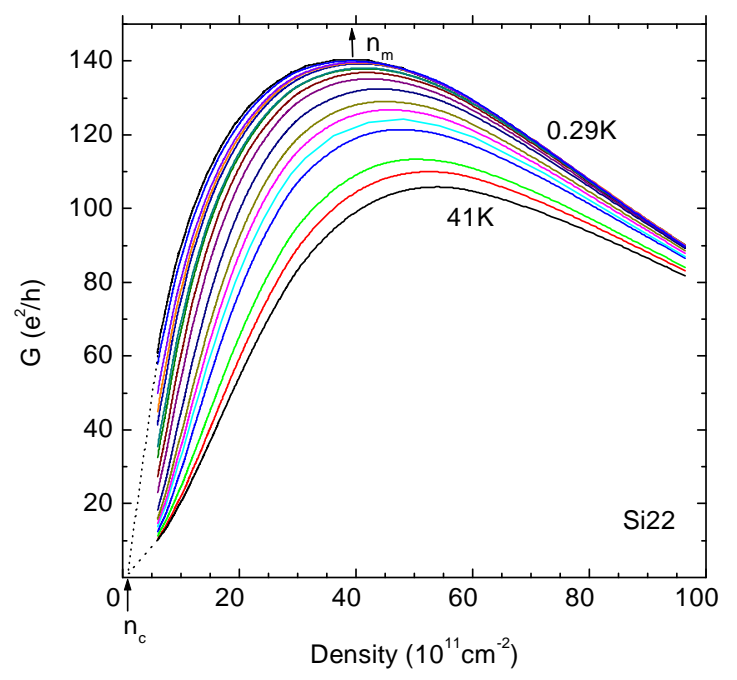

Fig1

Pudalov et al 


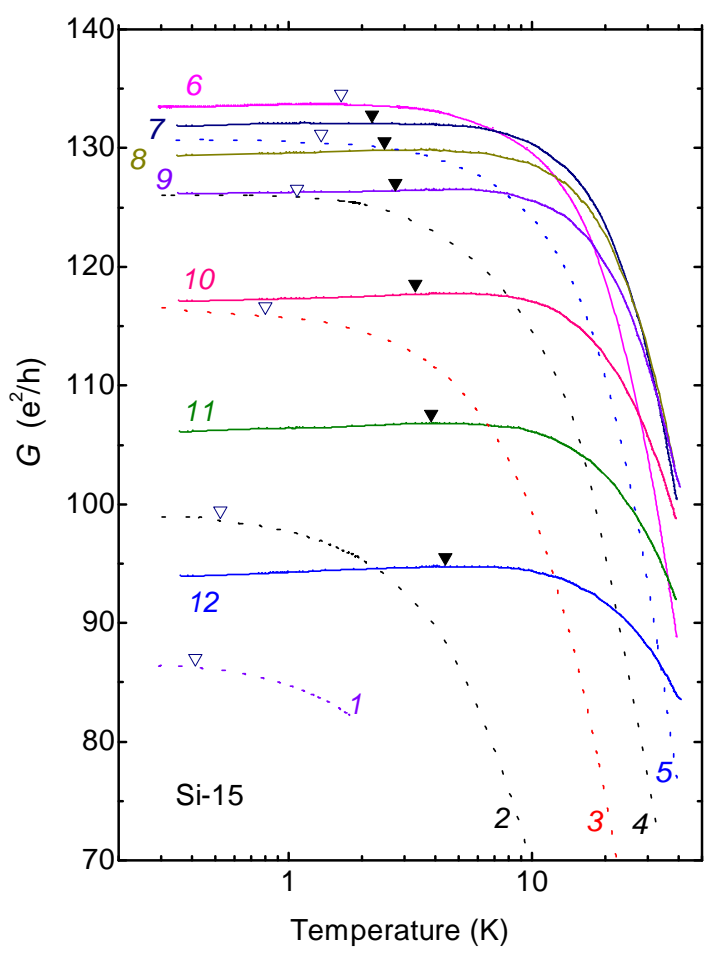

Fig. 2

Pudalov et al 


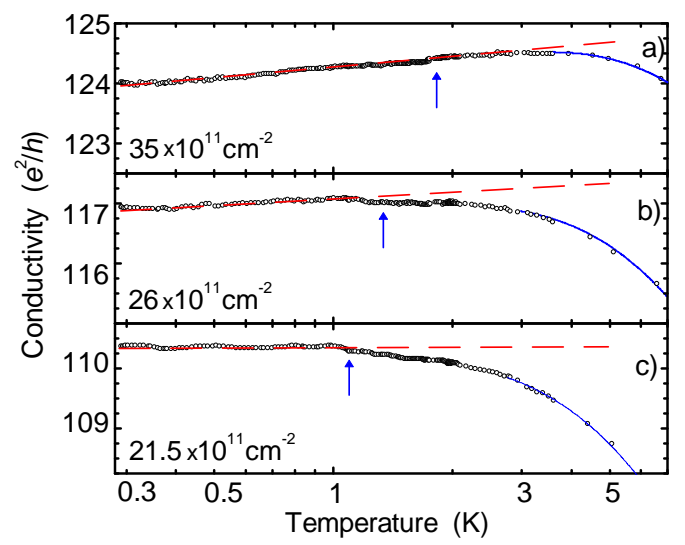

Fig.3
Pudalov et al. 